Tino Licht und Annelen Ottermann*

\title{
Aus der Frühzeit der Mainzer Skriptorien: Ein unbekanntes karolingisches Handschriftenfragment (Mainz, Wissenschaftliche Stadtbibliothek, Hs frag 20)
}

DOI 10.1515/bfp-2017-0004

Zusammenfassung: Die Überlieferung der frühen Mainzer Handschriften hat hohe Verluste verzeichnen müssen, weshalb der Wert der wenigen Fragmente aus Mainzer Bibliotheken erheblich ist. Anzuzeigen ist der Neufund eines karolingischen Fragments in der Wissenschaftlichen Stadtbibliothek Mainz, das den Matthäuskommentar des Hieronymus enthält und in das frühe IX. Jahrhundert datiert.

In Anbetracht ihrer Bedeutung wurde die Einbandmakulatur von ihrem Trägerband gelöst und in die noch junge Fragmentsammlung der Bibliothek aufgenommen. Ein solcher Eingriff in die historischen Befunde wird nur im Ausnahmefall vorgenommen und bedarf der besonderen Begründung.

Schlüsselwörter: Karolingische Kultur; Handschriftenfragment; Überlieferung

From the Beginning of Manuscript Culture in Mainz: An Unknown Carolingian Fragment (Mainz, Wiss. Stadtbibliothek, Hs frag 20)

\begin{abstract}
The transmission of mediaeval manuscripts from Mainz is weak, and therefore the rare fragments found in the Mainz libraries have an outstanding value as documents of the early manuscript culture. It has to be announced a new Carolingian fragment from the early $9^{\text {th }}$ century in Mainz Stadtbibliothek containing Jerome’s Commentary on Matthew.

Considering its importance, the waste paper was taken off the book it has covered and integrated into the new collection of fragments. Such a far-reaching decision should be taken only in exceptional cases like this.
\end{abstract}

Keywords: Carolingian culture; manuscript fragment; transmission

*Kontaktpersonen: PD Dr. Tino Licht, tino.licht@urz.uni-heidelberg.de

Dr.Annelen Ottermann, annelen.ottermann@stadt.mainz.de
Inhalt

1 Fragmente in Bibliotheken . . . . . . . . . . . 103

2 Fragment und Makulatur in der Stadtbibliothek Mainz ....................... 104

3 Hs frag 20: Fundsituation, Trägerband und Ablösung . . . . . . . . . . . . . . . . . . . 105

4 Zur Mainzer Paläografie im Allgemeinen . . . . . 106

5 Zu den Mainzer Skriptorien und ihren Einflüssen . 109

6 Zu Paläografie und Inhalt von Hs frag 20 der Stadtbibliothek Mainz . . . . . . . . . . . . . . . 111

\section{Fragmente in Bibliotheken ${ }^{1}$}

Fragmente, wie sie heute in Bibliotheken und Archiven auf uns kommen, sind immer das Ergebnis einer Überlieferungsstörung. ${ }^{2}$ Selbst den methodisch versierten Quellenforscher nötigen sie zu einer geschärften Wahrnehmung des in ihnen manifesten Überlieferungszufalls. Fragmente, an denen das Fragmentarische zwar buchstäblich greifbar, aber nicht unmittelbar begreifbar ist, stellen zwangsläufig auch im bibliothekarischen Alltag einen Störfall dar. Sie

„fordern heraus, bringen den Geschäftsgang aus dem alltäglichen Takt, werfen unzählige Fragen auf - dies gilt für ihre Auffindung, ihre Verzeichnung, ihre Erschließung, ihre Erhaltung, ihre Kontextualisierung, ihre Bekanntmachung und ihre dauerhafte Sichtbarmachung." ${ }^{3}$

Die Einheit von Trägerband und Makulatur aufzulösen, bedeutet zwangsläufig, die Fundsituation zu verändern. Unter Umständen heißt es auch, den Überlieferungsbefund in seinem Aussagewert für die buch- und rezeptions-

1 Annelen Ottermann verfasste Kapitel 1-3. Tino Licht ist Autor der Kapitel 4-6.

2 Vgl. zuletzt umfassend zum Thema ,Fragmente' den Sammelband von Neuheuser und Schmitz (2015), dessen Untertitel diesem Faktum Rechnung trägt.

3 Ottermann $(2015,148)$. 
geschichtliche Forschung zu schmälern oder ganz zu entwerten. Was wir vereinzeln, wird aus dem Kontext gerissen. Schlimmstenfalls ist dieser Zusammenhang endgültig verloren. Günstigenfalls lässt sich rekonstruieren, wie der einstmalige Kontext ausgesehen hat, durch Text- und Bilddokumentationen, Restaurierungsberichte und katalogtechnische Anmerkungen im OPAC.

Seit dem Beginn der Makulaturforschung vor fast 200 Jahren wurden von Bibliothekaren, Buch- und Einbandkundlern Fragmente abgelöst - durchaus nicht aus blindwütigem Sammel- und Entdeckungsfieber, sondern vor allem in ernsthaftem Forschungsdrang und der festen Überzeugung, für die Wissenschaft damit den einzig richtigen Schritt zu leisten. ${ }^{4}$ Die verheerenden Folgen für die spätere Forschung wurden von der Mehrheit nicht abgeschätzt, obwohl es auch immer Gegenstimmen gab, die für das Bewahren sprachen. Kaum ein Altbestandsbibliothekar wird nicht Entsprechendes in der von ihm betreuten Sammlung vorgefunden haben, und sogar bis in die jüngste Gegenwart wurde immer wieder die dem Zufall geschuldete Überlieferungsstörung früherer Jahrhunderte sehenden Auges ohne Not verlängert.

„Die Auffassung einer Einheit von Einband und Inhalt ist ein Produkt der neueren Wissenschaft. " ${ }^{5}$ Verantwortlich mit den exemplarspezifischen Evidenzen an frühneuzeitlichen Drucken umzugehen, impliziert heute, Fundsituationen $\mathrm{zu}$ konservieren und den Überlieferungszufall so weit wie möglich zu schützen. Es hat ein Paradigmenwechsel stattgefunden: wo immer es vertretbar ist, wird noch nicht abgelöste Makulatur in Gestalt von Einbänden, Deckelkonstruktionen und Falzverstärkungen in situ belassen, um aufsetzende überlieferungsgeschichtliche Forschung zu ermöglichen.

Wird von dieser Maxime abgewichen, so geschieht dies nur in begründeten Ausnahmefällen, in denen der zu erwartende Erkenntniswert aus der Erforschung eines voll umfänglich zugänglichen Fragments über den Ensemblegedanken gestellt wird. Die Verantwortung für oder gegen eine solch weitreichende Entscheidung müssen die Altbestandsbibliothekare übernehmen, sie unter Umständen

\footnotetext{
4 Haebler (1908, 541, 542) etwa war der festen Auffassung, „dass das Sammeln der Fragmente nur dann seine erschöpfende wissenschaftliche Bedeutung gewinnen kann, wenn es auf der breitesten Grundlage systematisch betrieben wird.“ In Entgegnung auf „diejenigen, die für das Belassen der Fragmente in den Einbänden eintreten“, hielt er es für „unbedingt wünschenswert“, dass „alle Bibliotheken angewiesen wären, auf alle solche Druck- und Handschriftenfragmente zu fahnden, [und] sie sorgfältig aus den Einbänden zu lösen“.

5 Oppitz (2002, 58). Unter der Kapitelüberschrift „Die Stecknadel im Heuhaufen oder Vom Nutzen der Fragmentforschung“ skizziert der Autor den Wandel im Umgang mit Fragmenten.
}

auch gegenüber gegenläufigen Interessen vertreten und transparent machen. Sie sind gut beraten, wenn sie zuvor fachwissenschaftliche Einschätzung eingeholt und sich im Kontakt mit Vertretern akademischer Einrichtungen über das Erkenntnisinteresse verständigt haben.

\section{Fragment und Makulatur in der Stadtbibliothek Mainz}

Die Rarasammlung der Wissenschaftlichen Stadtbibliothek Mainz wird seit ihrer Einrichtung Mitte der 1980erJahre laufend erweitert. Dies geschieht punktuell durch Zufallsentdeckungen aus der Alltagspraxis der Bereichsleitung und über Hinweise aus der Benutzung im weitesten Sinne. Vor allem aber basiert der Ausbau dieses Bereichs seltener und wertvoller Drucke des 16.-21. Jahrhunderts auf der systematischen Durchsicht des Magazinbestands. ${ }^{6}$ Sämtliche Exemplare, die mit Hand- oder Druckschriftenmakulatur eingebunden oder verstärkt sind, wurden bei dieser Durchsicht in die Rarasammlung aufgenommen, da die exemplarspezifischen Besonderheiten sie zu Unikaten hatten werden lassen. ${ }^{7}$ Bei der genaueren Sondierung dessen, was der globale ,Makulatur-Fischzug“ ergeben hatte, galt Zeugnissen mit deutschsprachigen oder hebräischen Fragmenten, illuminierten Texten und frühen Schriftbeispielen besonderes Augenmerk. Hier eröffnete sich ein weiter bibliothekarischer Handlungsspielraum zwischen Forschungsunterstützung und eigener Forschung in enger Vernetzung mit Vertretern der jeweiligen Wissenschaftsdisziplinen.

Die Grundsatzentscheidung, die Einheit von Trägerbänden einschließlich ihrer Provenienzmerkmale und der an oder in ihnen befindlichen Makulatur in aller Regel zu erhalten, war auch in Mainz gefallen. Sie gilt bis heute, doch ergab sich im Verlauf eines Vierteljahrhunderts mehrfach die fachliche Notwendigkeit, von dieser Maxime abzuweichen und handschriftliche Bruchstücke zu vereinzeln. Entsprechend verfahren wurde etwa bei BedaMakulatur aus dem Mainzer Skriptorium des 9. Jahrhunderts, ${ }^{8}$ Seiten aus Mainzer Rechnungsbüchern, ${ }^{9}$ einem Rennewart-Fragment, ${ }^{10}$ Teilen eines rabbinischen Mi-

6 Mit Stand vom 31.10.2016 umfasst das Raramagazin ca. 8160 Bände. Zu seiner Entwicklung vgl. die Begleitpublikation zur gleichnamigen Ausstellung von Ottermann (2008b).

7 Vgl. dazu Ottermann (2008a).

8 Heute Hs frag 1. Vgl. dazu Ottermann (1998).

9 Heute Hs frag 2. Vgl. dazu Dobras (2002).

10 Heute Hs frag 16. Vgl. dazu Ottermann und Klein (2008). 
drasch ${ }^{11}$ und im spektakulären Fall des spätkarolingischen Fragments eines illustrierten Apokalypsekommentars. ${ }^{12}$ Die wissenschaftliche Auswertung bildete in all diesen Fällen einen festen Bestandteil der Gesamtmaßnahme. Zur bibliothekarischen Selbstverpflichtung gehört die Verständigung auf größtmögliche Transparenz solcher Entscheidungen. In der Stadtbibliothek Mainz wird die virtuelle Zusammenführung von Trägerband und Fragment mit den Werkzeugen der Provenienzerschließung garantiert: katalogtechnisch durch eine Anmerkung bei den exemplarspezifischen Daten mit der standardisierten Formulierung „Ehemals Trägerband handschriftlicher Makulatur, jetzt unter Hs frag ...“.

\section{Hs frag 20: Fundsituation, Trägerband und Ablösung}

Von der Masse rarifizierter Druckwerke mit Makulatur aus lateinischen Liturgica des Spätmittelalters hob sich ein quartformatiger venezianischer Druck von 1563 hervor, der mit Bruchstücken aus dem Hieronymuskommentar zum Matthäusevangelium in karolingischer Makulatur vollflächig eingebunden war.

Autor des Trägerbandes ist der aus Granada stammende Dominikaner Jean Viguier, der 1527-1550 in Toulouse als Theologieprofessor lehrte. Seine Institutiones ad Christianam theologiam erschienen erstmals 1553 in Paris und sollten in den Folgejahren hier wie auch in Venedig und Köln rasch weitere Auflagen erfahren. Die vorliegende Ausgabe wurde $1563^{13}$ in der Werkstatt des italienischen Druckers Francesco Lorenzini fertiggestellt. ${ }^{14}$

Der Erstbesitzer des Mainzer Exemplars lässt sich nicht mehr feststellen, da sein Provenienzeintrag geschwärzt ist und nur noch fragmentarische Informationen preisgibt: Demnach scheint es sich um einen Ordensangehörigen zu handeln, der 1580 (1586?) seine temporäre Nutzung an dem theologischen Lehrwerk mit der Formel $\mathrm{Ad}$ usum einleitete. Eine zweite Schicht in der Provenienzkette eröffnet sich mit einem gut erhaltenen Vermerk auf dem fliegenden Blatt: Durch die Floskel iure merito possidet me dokumentierte hier der aus Stadt oder Bistum Speyer stammende Geistliche Andreas Maijer 1627 den rechtlich abge-

11 Etwa Hs frag 15. Vgl. dazu Lehnardt und Ottermann (2015, 145155).

12 Heute Hs frag 18. Vgl. dazu Ottermann (2014).

13 Viguer (1563).

14 Lorenzini ist für die Jahre 1547-1581 nachgewiesen. Er druckte vor allem in Venedig und verlegte seine Offizin 1581/82 nach Turin. sicherten und unbedenklichen Besitzübergang an ihn. Nach eigenem Bekunden war er zu diesem Zeitpunkt Vicarius senior am Speyerer Kollegiatstift St. German und Moritz. ${ }^{15}$ Von der Hand Mayers, der intensiv mit dem Exemplar gearbeitet haben muss, stammen eindeutig die zahlreichen Marginalien im Text und auch die handschriftlichen Textergänzungen in bräunlicher Tinte auf dem beschädigten Titelblatt. Möglicherweise gelangte das Exemplar von ihm zu einem Angehörigen des Speyerer Karmelitenkonvents und von dort in die Mainzer Karmelitenbibliothek, ${ }^{16}$ deren Bibliothekar den Besitz und eine Vielzahl von Signaturen eintrug.

Es ist davon auszugehen, dass der Erstbesitzer des Jahres 1580/86 das Werk mehr als 10 Jahre nach seiner Drucklegung erwarb und den Einband des dickleibigen Werkes veranlasste. Die bruchstückhaften Angaben zu seiner Person geben keinerlei Anhaltspunkte für die im Kontext dieses Beitrags vorrangig interessierenden Fragen: An welchem Ort stand die für den Einband verwendete Makulatur einem Buchbinder zur Verfügung, wie gelangte sie dorthin, wann und wo war die Makulierung der karolingischen Handschrift erfolgt?

Dass die Entdeckung des karolingischen Fragments nähere Untersuchung im Hinblick auf Datierung und Schriftheimat verdiente, war rasch deutlich. Die Ablösung der Makulatur und ihre Aufnahme in die im Aufbau befindliche Fragmentsammlung erfolgte $1998 .{ }^{17}$ Erst Jahre später allerdings wurde der Kontakt zum Mittellateinischen Seminar der Heidelberger Ruprecht-Karls-Universität hergestellt. Walter Berschin und Tino Licht bewerteten das Stück 2012 als „qualitätvolles karolingisches Fragment“ und urteilten bei dieser ersten Kenntnisnahme: „Insgesamt ergibt sich eine Lokalisierung in das Moseloder Mittelrheingebiet; Mainz ist nicht ausgeschlossen." ${ }^{\text {"18 }}$

15 In den Referenzwerken ist Maijer (Mayer) nicht nachweisbar. Cf.u. a. Engels (2009).

16 Zu dieser Provenienz vgl. Ottermann (2016).

17 Das Fragment trägt seitdem die Signatur Hs frag 20. Seine Erschließung wie die aller übrigen bisher nur intern verzeichneten Fragmente ist in Arbeit. Die Ergebnisse sollen 2017 in Manuscripta mediaevalia zugänglich gemacht werden. Die verstärkte Erschließungsarbeit steht im Zusammenhang mit einer für 2018 geplanten Buchpublikation unter dem Titel „Unser Wissen ist Stückwerk“. Der ,entkleidete“ Buchblock erhielt 1998 einen Konservierungseinband aus Pergament. 18 Brief vom 21.12.2012 an die Bereichsleiterin. 


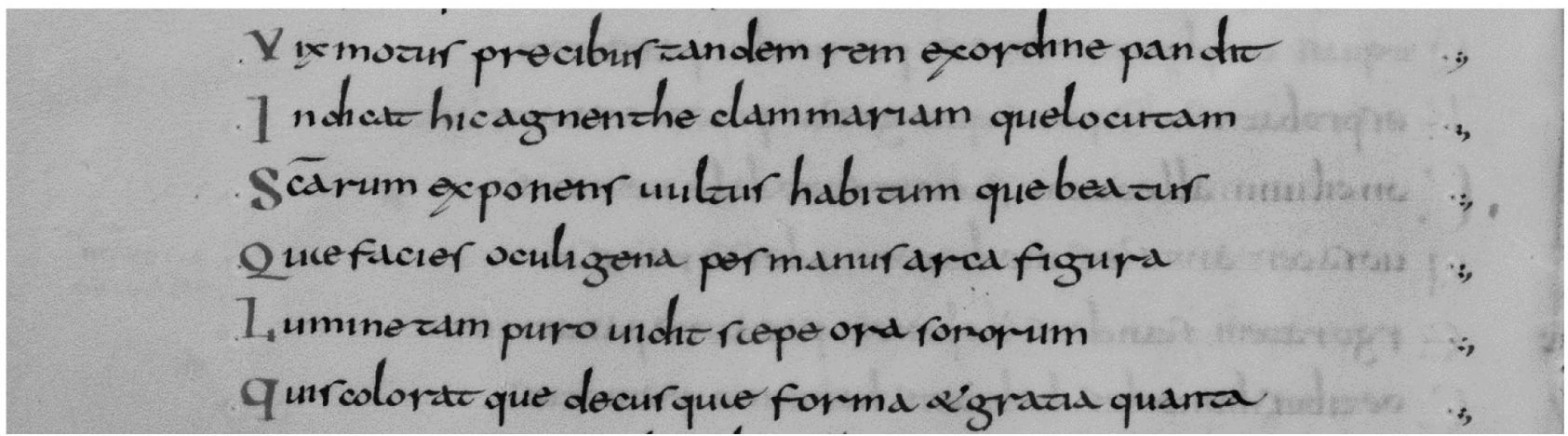

Abb. 1: Rom, Biblioteca Apostolica Vaticana, Pal. lat. 845, fol. 171, Ausschnitt (»Martinellus«; Mainz, 1. Viertel des IX. Jahrhunderts). Der Codex gehört zu den ältesten Handschriften in karolingischer Minuskel aus Mainz und lässt den kantigen, spitzen und rechtwinkligen Mainzer Stil gut erkennen, zu dem auch das gebrochene t gehört (motus Z. 1). Im vorderen Teil (bis ca. fol. 59) ist die Schrift noch insular geprägt. Das Auszeichnungsalphabet ist ein Mischalphabet (Capitalis-V und - $Q$ ).

\section{Zur Mainzer Paläografie im Allgemeinen}

Die Mainzer Skriptorien beginnen - soweit man das angesichts der großen Verluste rekonstruieren kann ${ }^{19}$ - vor 800 mit der Handschriftenherstellung in insularer Schrift. ${ }^{20}$ Dieser insulare Einfluss ist generell ein Phänomen des deutsch-insularen Gebietes und betrifft in der frühen Karolingerzeit etwa auch das nahegelegene Kloster Lorsch. ${ }^{21} \mathrm{Er}$ ist angesichts der Vorprägung durch Bonifatius und Lull und angesichts der Mainzer (kulturellen) Nähe zu Fulda, wo man bis in die Mitte des IX. Jahrhunderts für Hausexemplare insulare Schrift verwendet, ${ }^{22}$ vorgegeben und nicht überraschend. Das Insulare in den Mainzer Skriptorien interessiert hier nur soweit, als es einen stilistischen Einfluss auf die Mainzer Handschriften ausübt, auf ihre Initialen, ihre Auszeichnungsschriften und auf den Stil der Hauptschrift selbst. Um mit Letzterer zu beginnen: Als die Mainzer Skriptorien in den ersten Jahrzehnten des IX. Jahrhunderts zur karolingischen Minuskel übergehen, nimmt die Schrift einen kantigen, spitzen und rechtwinkligen Stil an, sie zeigt „aufrechte Schreibweise“, „knickt scharf ab“.23

19 Den ersten Versuch einer paläografischen Studie zu den Skriptorien im karolingischen Mainz unternahmen Lindsay und Lehmann (1925). Eine aktuellere Liste von karolingischen Handschriften aus Mainz gibt Hanselmann (1987, 86 f.).

20 Wichtigster Zeuge dieser insularen Frühphase in Mainz ist die auf Oxford, Bodleian Library, Laud. misc. 263 und zwei Fragmente in Würzburg, Universitätsbibliothek, M.p.j.f. 7 + M.p.th.f. 28 verteilte Handschrift mit Gregors Regula pastoralis, in welcher der Colophon eines Willibaldus diaconus eingetragen ist. Vgl. Lowe (1959).

21 Vgl. Becker und Licht $(2016,6)$.

22 Licht (2015).

23 Ottermann $(1998,302)$.
Das ist Ausdruck des angesprochenen insularen Beginns und der Weiterführung insularer Traditionen. Der aufgrund seiner Paläografie als besonders alt erkennbare ,Mainzer Martinellus' in Rom, Biblioteca Apostolica Vaticana, Pal. lat. 845 ist ein Codex, an dem man gut den Übergang vom insularen zum karolingischen Schriftgebrauch beobachten kann: Nach dem ersten Viertel der Handschrift (fol. 59 $)$ gehen die Schreiber vom halbunzialen $g$ der insularen Schrift zum geschlossenen g der karolingischen Minuskel über (Abb. 1). Stilmerkmal der karolingischen Minuskel aus den Mainzer Skriptorien ist es, „die verbreiterten Oberlängen spitz nach rechts auszuziehen“ ${ }^{24}$ oder mit anderen Worten „die verstärkten Oberlängen schräg abzuschneiden“. ${ }^{25}$ Bei Schreibern, die den Mainzer Stil mit sicherer Hand mustergültig zu schreiben verstehen, wirken die Schäfte wie aufgerichtete Skalpelle. Für einige Buchstaben oder Buchstabenkombinationen ergeben sich weitere markante Formen: Das $t$ ist im unteren, eigentlich runden Teil gern eckig gebrochen (Abb. 1) und steht manchmal sogar rechtwinklig auf der Grundlinie. Das unziale a präsentiert sich - für eine karolingische Minuskel des IX. Jahrhunderts ist das ungewöhnlich - bisweilen fast aufgerichtet (Abb. 2). Die weit nach unten gezogenen Rechtsschrägen des x erzeugen ein markantes Schriftbild (Abb. 3). Eine spezifische Gewohnheit, die nicht durch insularen Einfluss erklärt werden kann, dürften die untergestellten Doppel-s sein (Abb. 2).

Leider verwenden nicht alle Mainzer karolingischen Schreiber dieses untergestellte Doppel-s. Es wäre sonst eine Ohrmarke der Mainzer Skriptorien. Neben den Charakteristika der Hauptschrift - Skalpellschäfte, eckig gebrochene t,

24 Bischoff (1998) Nr. 842. 25 Bischoff (1998) Nr. 1754. 


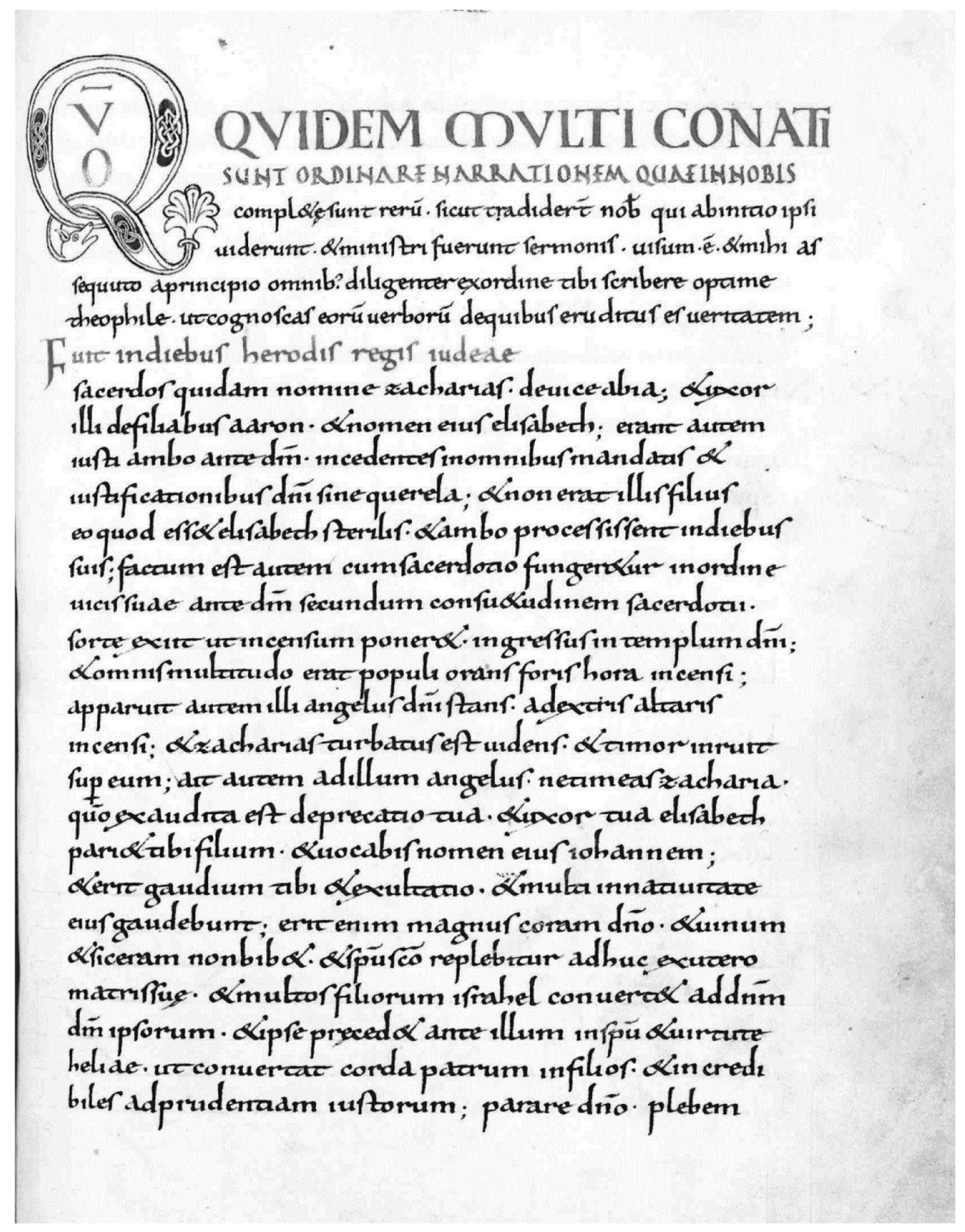

Abb. 2: Zeitz, Stiftsbibliothek, $2^{\circ}$ Ms. perg. lat. 5, fol. $69^{r}$ (Evangeliar; Mainz, 2. Viertel des IX. Jahrhunderts). Eine der zahlreichen in Mainz hergestellten Evangelienhandschriften. Im Tierkopfmotiv der Initiale manifestiert sich insulare Tradition. Im Skriptorium wird arbeitsteilig gearbeitet: Ein Vorschreiber hat die Auszeichnungszeilen bis zur Rubrik Fuit in diebus ... vorgeschrieben, danach setzt der Hauptschreiber ein, der mustergültig die Mainzer Skalpellschäfte, das beinahe aufrechte a und das untergestellte Doppel-s (processissent Z. 12) demonstriert. ${ }^{26}$

untergestellte Doppel-s - weisen auch die Mainzer Auszeichnungsschriften markante Eigenheiten auf. Hauptauszeichnungsschrift ist eine rote Unziale (schwarz bevorzugt in den Seitentiteln), die bei frühen Handschriften eher noch

26 Das Zeitzer Evangeliar blieb in der Literatur zur Mainzer Schriftgeschichte bisher unbeachtet und wurde von Herrad Spilling und Hartmut Hoffmann aufgrund von Abbildungen datiert und lokalisiert. Vgl. Bulitta (2006, 70 Anm. 15). ein Mischalphabet mit vielen Capitalisbuchstaben (z. B. E, $\mathrm{Q}$ und V) ist (Abb. 1 und 3). Schon da aber fällt als Kennbuchstabe das A mit dem extrem engen, spitzwinkligen oder gar nur als Strich ausgeführten Auge auf (Abb. 3).

Einen eigenen Charakter verleihen der Auszeichnungsschrift die breit gedeckten Schäfte von H und L. Höherrangige Schriften werden in Mainz sparsam verwendet. Capitalis rustica bleibt selten. Wenn die in der Schrifthierarchie an oberster Stelle eingeordnete Capitalis quadrata 


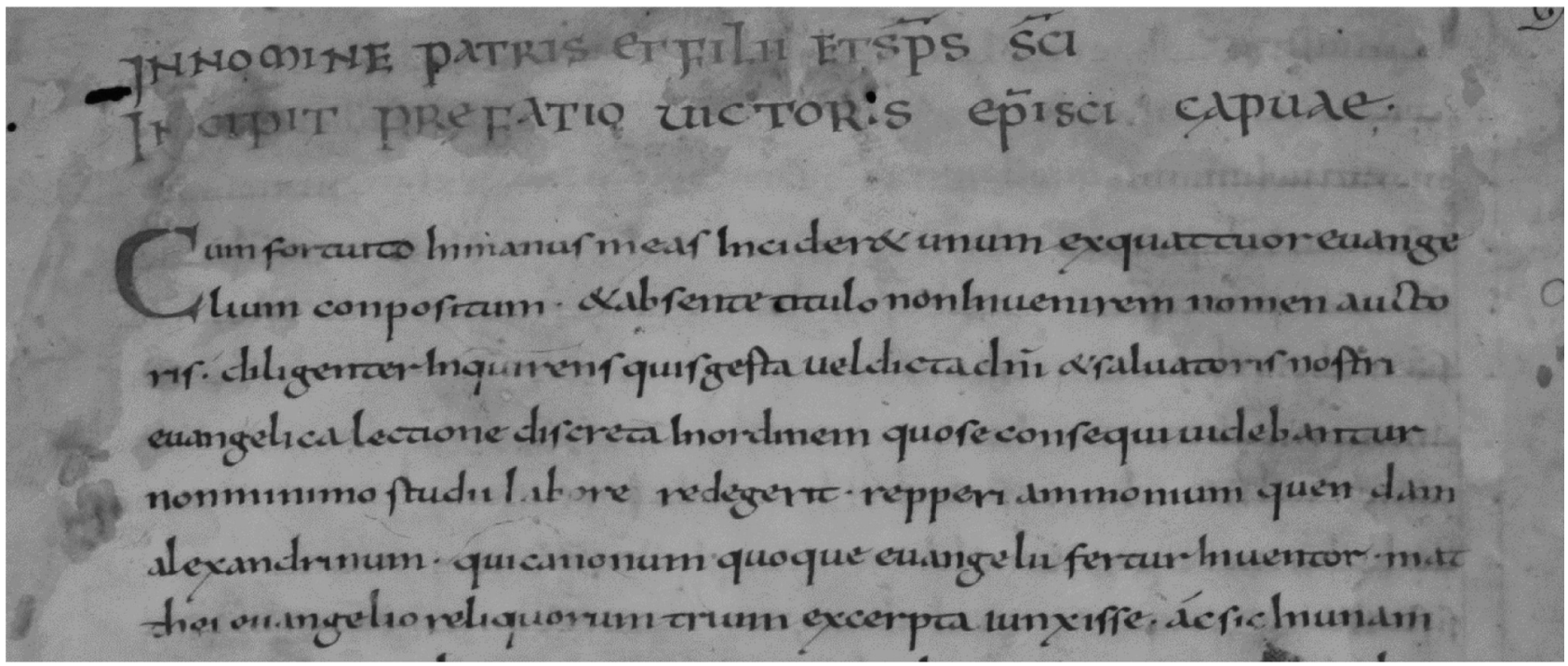

Abb. 3: St. Gallen, Stiftsbibliothek, 56, p. 1 (»Lateinisch-althochdeutscher Tatian«; Mainz, 2. Viertel des IX. Jahrhunderts). Mainzer Gewohnheit zeigt sich im kantigen, scharf geschnittenen Stil, den Skalpellschäften, den weit nach unten gezogenen Rechtsschrägen des $x$ und dem untergestellten Doppel-s (z. B. iunxisse letzte Zeile). Die Auszeichnungszeilen im Mischalphabet lassen gut das enge, spitzwinklige Auge des unzialen A erkennen.

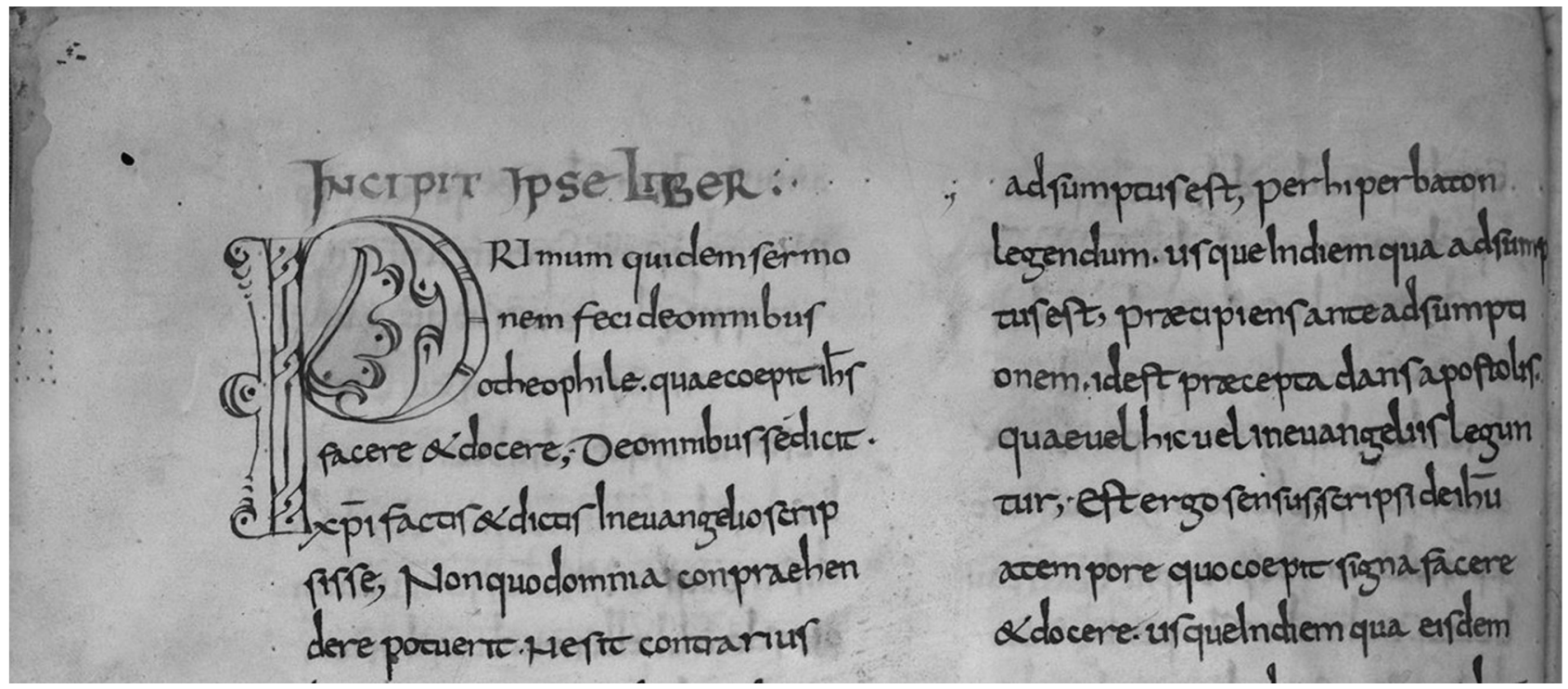

Abb. 4: Gotha, Forschungsbibliothek, Memb. I 45, fol. 2v , Ausschnitt (Beda, Commentarii; Mainz, 1. Drittel des IX. Jahrhunderts). In der P. Initiale ist das ,Mainzer Leitmotiv', die dornartige Bildung am Blattansatz erkennbar. ${ }^{27}$

verwendet wird, zeigt sie neben den vertrauten runden Formen eckige $\mathrm{C}$ und $\mathrm{G}$. Auch die Mainzer Initialen weisen Eigenheiten auf. Ein Merkmal, das sich auch andernorts finden lässt, sind die aus insularer Tradition erklärbaren

27 Bischoff (2004) Nr. II, 1420 datiert auf „IX. Jh. ca. Mitte“, allerdings ist keine ur-Kürzung verwendet, und auch sonst wirkt die Schrift (etwa durch Reste von Decrescendo) älter.
Tierköpfe als Ablaufmotiv (Abb. 2). Spezifischer ist eine Blattform, die zum Mainzer Leitmotiv erklärt worden ist: Ihr Kennzeichen ist die „dornartige Bildung am Blattansatz“ (Abb. 4). ${ }^{28}$ Alle angegebenen Schriftmerkmale bleiben ohne Beweiskraft, wenn sie nur als Einzelerscheinung auftreten, und erst ihre Kombination liefert einen

28 Bierbrauer (1997, 563). Die Beobachtung fußt auf Weiner (1992). 
Hinweis auf Mainzer Schriftheimat. Umgekehrt stellt das Fehlen des einen oder anderen Kennzeichens kein Ausschlusskriterium dar.

\section{Zu den Mainzer Skriptorien und ihren Einflüssen}

Die Mainzer Schriftgeschichte ist nicht allein wegen der hohen Überlieferungsverluste schwierig zu rekonstruieren. Die Verhältnisse komplizieren sich noch dadurch, dass wir zahlreiche Handschriften von ausgesprochenem Mischcharakter haben: In die karolingische Minuskel im charakteristischen Mainzer Stil mischen sich immer wieder Hände, die in anderen Schreibschulen ausgebildet worden sind oder den kantigen Mainzer Stil nicht voll adaptieren. Bisher war außerdem immer von ,Mainzer Skriptorien“ die Rede, denn ein exklusiver Ort für die dortige Handschriftenproduktion ist nicht ermittelt. Monastische Konvente, allen voran St. Alban, kommen in Betracht, auch eine Mainzer Domschule kann man sich gut vorstellen. Mit Gewissheit nach St. Alban ist der nicht sehr umfangreiche komputistische Codex Rom, Biblioteca Apostolica Vaticana, Pal. lat. $1447 \mathrm{zu}$ lokalisieren, in dem bei der Anlage des Kalendars prominent die DEDICATIO BASILICAE SANCTI ALBANI MARTYRIS (fol. $17^{\mathrm{v}}$; 1 . Dezember) eingetragen worden ist, und zwar in der für Mainz charakteristischen Unziale mit dem engen, im Strich auslaufenden Auge von a. Der Codex ist jenseits der Lokalisierung für die Mainzer Schriftgeschichte auch deshalb von herausragender Bedeutung, weil sich auf fol. $19^{\mathrm{r}}$ ein im Eindruck der Ereignisse vorgenommener Nachtrag zum Wechsel von Richulf zu Haistulf auf dem Mainzer Erzbischofsstuhl findet. ${ }^{29}$ Für das Manuskript ergibt sich daraus ein Terminus ante quem im Jahr des Bischofswechsels 813. Aus St. Alban stammt folglich das älteste erhaltene, datierbare Mainzer Manuskript in karolingischer Minuskel.

Einen weiteren Fixpunkt unter den Mainzer Skriptorien bildet ein Gelehrten- und Schülerkreis um den Iren Probus, der ein intellektuelles Schwergewicht in der ersten Hälfte des IX. Jahrhunderts gewesen zu sein scheint. Bei seinem Tod am 25. Juni 859 erging ein hymnischer Nachruf: „Wie bescheiden und klug, geduldig und keusch er gewesen, / können die Buchstaben nicht, kann keine Zunge erzähln“. ${ }^{30}$ Zwei Handschriften aus seinem Umkreis sind er-

29 Bischoff (1981, 104f.).

30 Quam prudens, humilis, patiens castusque fuisset, / littera vel lingua nulla referre potest. Kurze (1891, 54). halten. Die Zuordnung ist nur vermutungsweise doch hinreichend plausibel vorgenommen worden, weil in den Handschriften irische Glossen auftauchen. ${ }^{31}$ Das Manuskript Berlin, Staatsbibliothek Preußischer Kulturbesitz, Ms. theol. lat. quart. 690 ist dabei so inhomogen, vereint Mainzer, französische und insulare Hände, dass man es sich am besten ,in einer von Schülern sehr verschiedener Herkunft besuchten Schule vorstellt, die sich in Mainz befand. “32

Aus Mainz ist ferner ein Augustinuscodex (heute München, Bayerische Staatsbibliothek, Clm 8107) erhalten, aus dem sich eine Anekdote der frühen Mainzer Bestandsgeschichte erhellt. Er ist seiner Paläografie nach in Mainz im zweiten Viertel des IX. Jahrhunderts entstanden und gehört gleichfalls zu den Handschriften, für die Mainzer Schriftheimat gesichert ist, obwohl eine Hand (am Anfang) gar nicht die typischen Mainzer Skalpellschäfte erkennen lässt. ${ }^{33}$ Das Manuskript hat die Retractationes des Augustinus zum Inhalt, jenes Opus also, in dem der Kirchenvater am Ende seines Lebens die Werke aus eigener Feder verzeichnet und kritisch rezensiert hat. Das vorangestellte Inhaltsverzeichnis mit der Werkliste des Augustinus ist von einer Hand des IX. oder X. Jahrhunderts durchgearbeitet worden, wobei alle in der Bibliothek vorhandenen $\mathrm{Au}$ gustinusschriften mit einem $\mathrm{h}$ (=habemus) markiert worden sind. In drei Fällen waren die verzeichneten Werke nicht mehr in der Bibliothek, weil man sie entwendet hatte. ${ }^{34}$ Neben den Titeln (auf fol. $1^{\mathrm{v}}$ ) hat der Bibliothekar mit einem Fingerzeig auf die Herkunft des Gauners vermerkt: iste / et iste /et iste sublati sunt a Fuldensi latrone. ${ }^{35}$ An welcher Bibliothek hat sich der Dieb aus Fulda bedient? Wahrscheinlich an der Dombibliothek, denn der Codex befand sich laut Eintrag von 1479 zumindest im späten Mittelalter daselbst.

Sehr gut in den Kontext eines Skriptoriums an der Domschule könnte auch ein ,Mainzer Original' passen, von dem wir den karolingischen Besitzer kennen, den Mainzer Chorbischof Thiotmar. ${ }^{36}$ Das Amt des Chorbischofs hatte in der Westkirche seinen Höhepunkt in der Karolingerzeit. Ihm waren Befugnisse übertragen, die sonst nur der Bischof innehatte, und seine Kernaufgabe bestand in der Entlastung des Bischofs gerade bei den zahlreichen Weihehandlungen. Der Chorepiskopat war ei-

\footnotetext{
31 Bischoff $(1981,42)$.

32 Bischoff $(1981,43)$.

33 Bischoff (2004) Nr. 3096.

34 Lindsay und Lehmann (1925, 28).

35 Dieses und dieses und dieses [Buch] sind von einem Dieb aus Fulda gestohlen worden.

36 Vgl. zu ihm Gottlob (1928, 32 f.).
} 


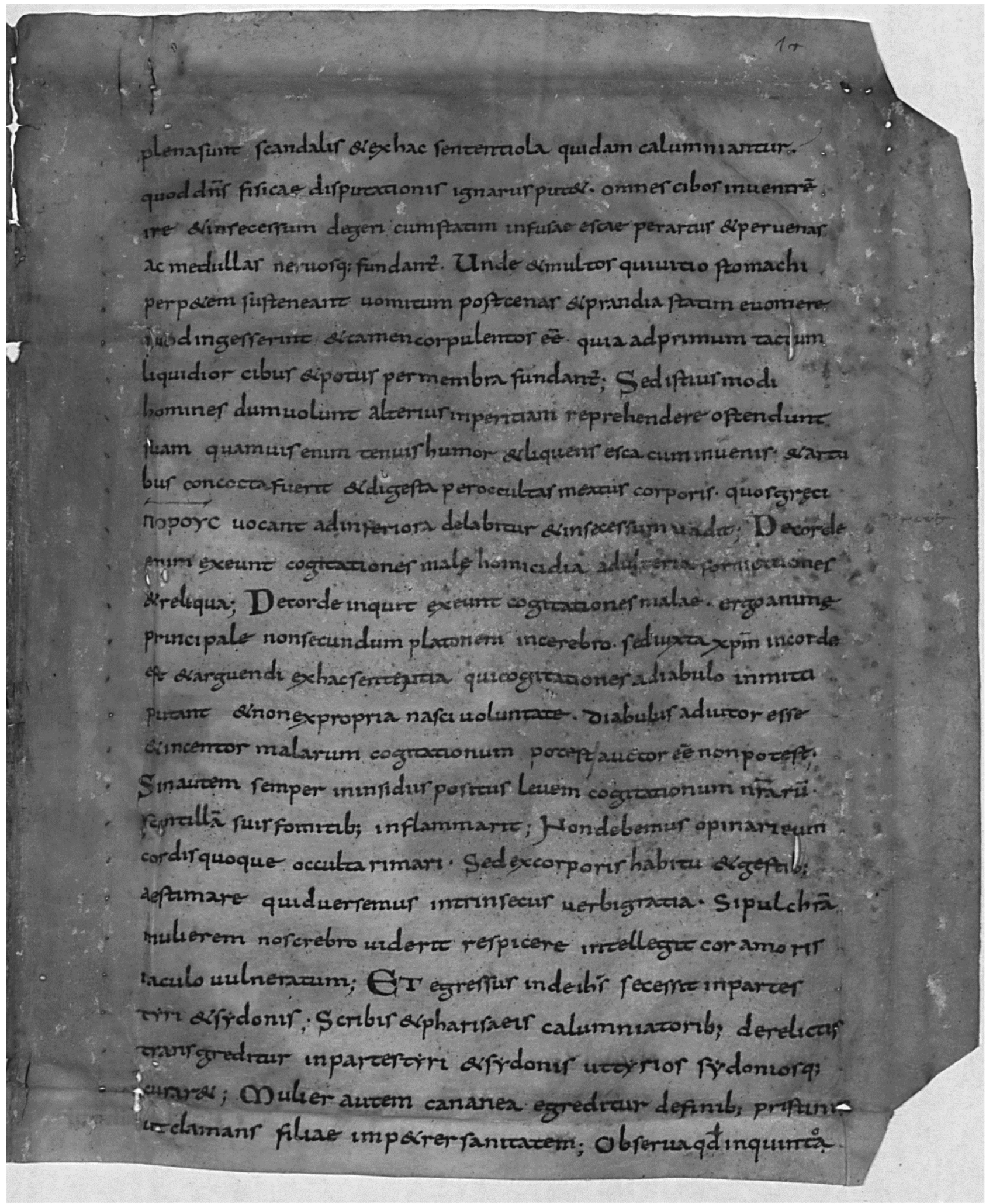

Abb. 5: Mainz, Wiss. Stadtbibliothek, Hs frag 20, fol. $1^{r}$ (Hieronymus, Commentarii in evangelium Matthaei; Mainz, 1. Drittel des IX. Jahrhunderts). Hand 1 schreibt eine runde Minuskel ohne Skalpellschäfte. Auffällig ist das leicht rückwärts geneigte unziale E (Et fünftletzte Zeile). Zur Abkürzung von -ur wird noch immer die us-Kürzung verwendet (fundantur Z. 4). 


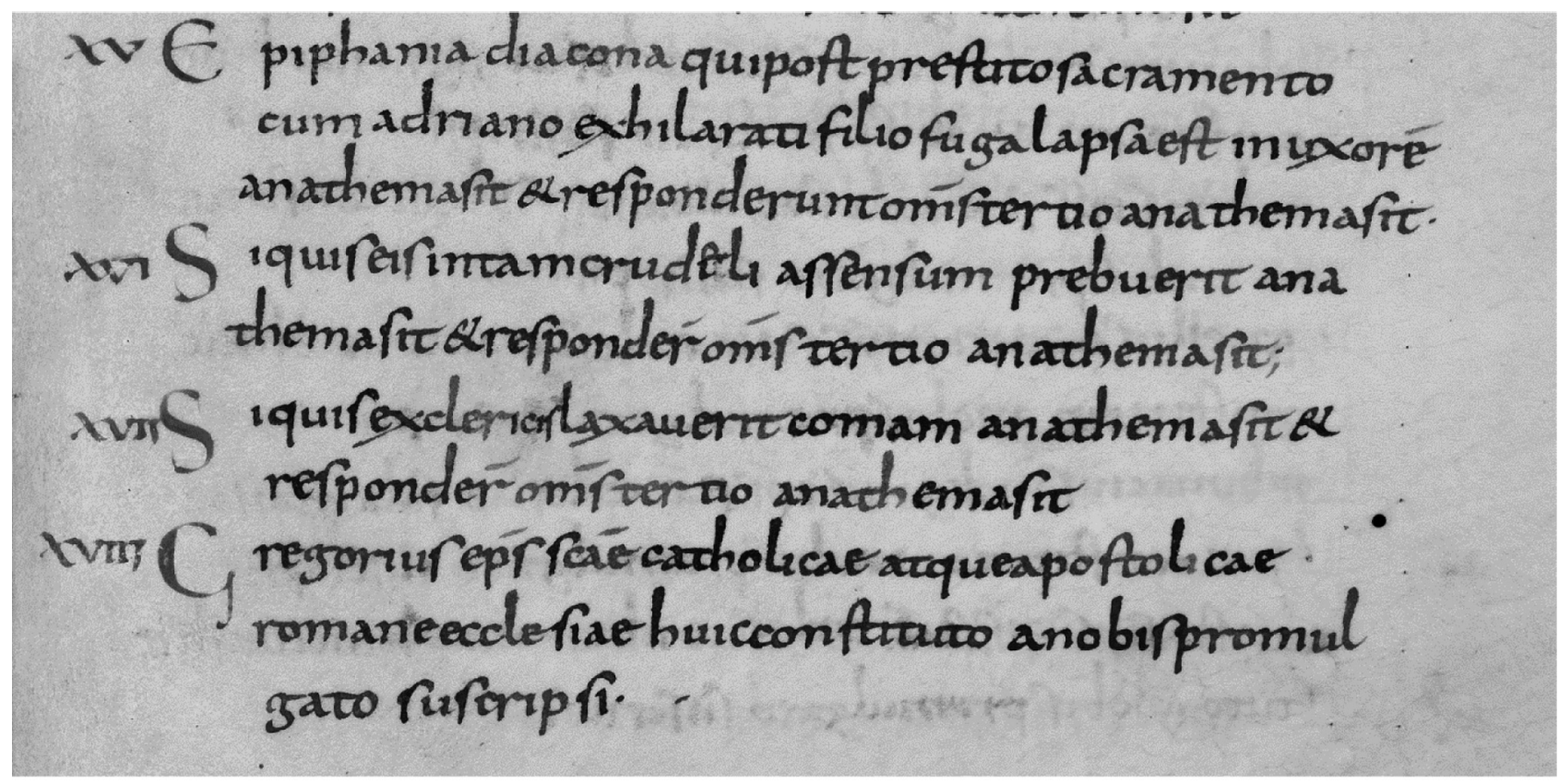

Abb. 6: Rom, Biblioteca Apostolica Vaticana, Pal. lat. 578, fol. 122 ${ }^{r}$ (Decreta pontificum; Mainz, 1. Hälfte des IX. Jahrhunderts). Runde Minuskel ohne Skalpellschäfte. In der Auszeichnungsschrift taucht das leicht rückwärts geneigte unziale E auf (auch die weiteren Auszeichnungsbuchstaben zeigen Linksneigung).

ne umstrittene Institution, weshalb der Mainzer Erzbischof Hrabanus Maurus (†856) eigens einen offenen Brief zur Rechtfertigung der Chorbischöfe geschrieben und zwischen 830 und 842 an Bischof Drogo von Metz (†855) gesandt hat. ${ }^{37}$ Auf lange Sicht hatte dieses Rechtfertigungsschreiben keinen Erfolg. Die Chorbischöfe verschwinden am Ende des Frühmittelalters fast ganz aus den westlichen Kathedralen. ${ }^{38}$ Chorbischof Thiotmar von Mainz war Mitte des IX. Jahrhunderts an der Mainzer Kathedrale der Amtsgehilfe des Hrabanus Maurus. Aus Thiotmars Besitz ist der Codex Paris, Bibliothèque Nationale, lat. 2443 erhalten, der in der typischen karolingischen Minuskel im Mainzer Stil steht und nur 29 Blätter umfasst. ${ }^{39}$ Sein Inhalt sind zwei Briefe des Hrabanus Maurus, zuerst der offene Brief zur Verteidigung des Chorepiskopats, danach ein brisantes Mahnschreiben an Kaiser Ludwig den Frommen (†840) über den rechten Umgang mit den Söhnen.$^{40}$ Der wohl autografe Besitzeintrag Thiotmars auf fol. $1^{\mathrm{r}}$ lautet: Thiotmar corepiscopus adquisivit. ${ }^{41} \mathrm{Am}$ ehesten wird er ein solches ,episkopales Manuskript‘ am Skriptorium der Domschule in Auftrag gegeben haben.

37 Vgl. dazu die Ausgabe von Maurus und Dümmler (1899, 431-439). 38 Gottlob (1928, 135-145).

39 Bischoff (2014) 41-94.

40 Ausgabe Maurus und Dümmler (1899, 403-415).

41 Chorbischof Thiotmar hat [den Codex] erworben.
Gesichert ist für die Handschrift die Entstehung vor oder im Jahr 857, dem Todesjahr des Thiotmar, und in diese Datierung in die Mitte des IX. Jahrhunderts fügt sich gut, dass darin die ur-Kürzung sicher verwendet wird. Im Zusammenhang mit Hrabanus Maurus sei abschließend noch auf die Nähe einiger Mainzer Manuskripte zur Handschriftenproduktion in Fulda hingewiesen. Herausragendes Zeugnis dieser Nähe ist die als Meilenstein deutscher Sprach- und Literaturgeschichte bekannte, lateinisch-althochdeutsche Evangelienharmonie, die heute in St. Gallen, Stiftsbibliothek, 56 aufbewahrt wird (vgl. Abb. 3). Dieser »Lateinisch-althochdeutsche Tatian« steht bis p. 24 in der Schrift von Mainz, danach in der Schrift von Fulda und hat damit wohl zwei Orte zur Schriftheimat. ${ }^{42}$

\section{$6 \mathrm{Zu}$ Paläografie und Inhalt von Hs frag $\mathbf{2 0}$ der Stadtbibliothek Mainz}

Das Doppelblatt Hs frag 20 der Stadtbibliothek Mainz zeigt das Nebeneinander zweier Hände, von denen Hand 1 (fol. 1) die Mainzer Schriftmerkmale zurückhaltend, Hand 2 (fol. 2) deutlich demonstriert. Nach Mainz passen

$42 \operatorname{Bischoff}(1981,78$ f.). 


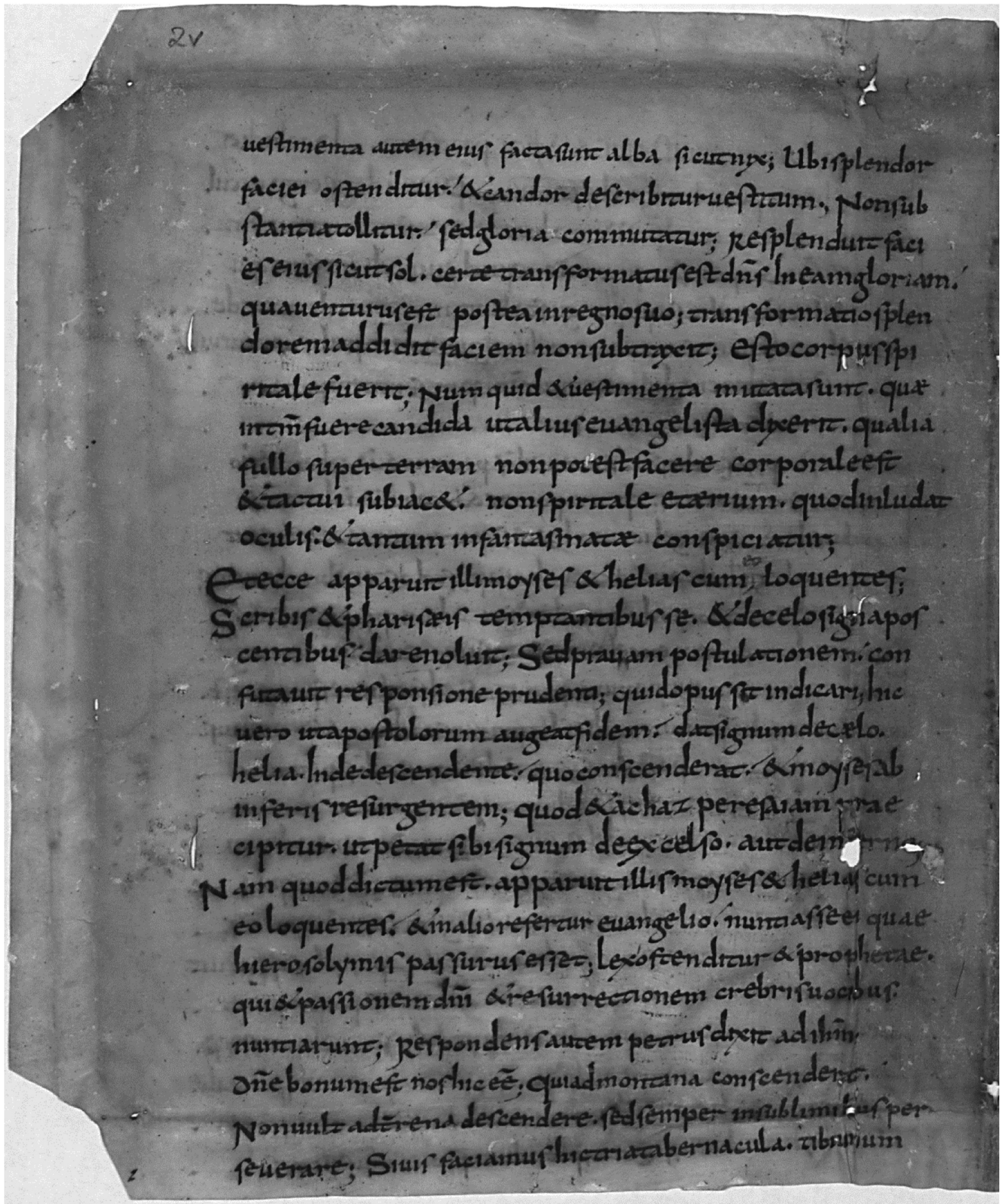

Abb. 7: Mainz, Wiss. Stadtbibliothek, Hs frag 20, fol. 2v (Hieronymus, Commentarii in evangelium Matthaei; Mainz, 1. Drittel des IX. Jahrhunderts). Hand 2 demonstriert den Mainzer Stil der karolingischen Minuskel mit den charakteristischen Skalpellschäften, den weit nach unten gezogenen Rechtsschrägen des x und den untergestellten Doppel-s (passionem fünftletzte Zeile). Ein individuelles Merkmal scheint das y mit der beinahe aufgerichteten Rechtsschräge zu sein (Moyses Z. 12). 
bei Hand 1 (Abb. 5) die weit nach unten gezogenen Rechtsschrägen des x, es fehlen die typischen Mainzer Skalpellschäfte, das unten eckig gebrochene $t$ und die untergestellten Doppel-s. Insgesamt wirkt die Hand gedrungener als die konventionellen Mainzer Hände. Dafür passen die von ihr zur Interpunktion und Gliederung verwendeten Auszeichnungsbuchstaben gut ins Bild der Mainzer Überlieferung, etwa das leicht, zurückgelehnte' unziale E, wie es auch im Codex Rom, Biblioteca Apostolica Vaticana, Pal. lat. $578 \mathrm{zu}$ beobachten ist (Abb. 6), oder ein altertümliches Capitalis-A mit gebrochenen Mittelbalken (das sich z. B. auch in der Handschrift Paris, Bibliothèque Nationale, lat. 7587 , fol. $74^{\mathrm{r}}$ finden lässt).

Hand 2 ist eine mustergültige Mainzer Hand und vereint alle Schriftmerkmale der karolingischen Minuskel am Ort: Skalpellschäfte, untergestelltes Doppel-s, eckig gebrochenes $t$ und weit nach unten gezogene Rechtsschräge des $\mathrm{x}$. Wollte man ein individuelles Charakteristikum der Hand benennen, käme das unpunktierte y in Betracht, dessen Rechtsschräge fast aufgerichtet ist.

Inhalt des Doppelblattes ist der beliebte und verbreitete Kommentar des Hieronymus zum Matthäusevangelium. Da zwischen dem ersten und zweiten Blatt eine erhebliche Textlücke klafft, gehörte die Doppelseite in den äußeren Teil der ursprünglichen Lage. Der Matthäuskommentar des Hieronymus ist so etwas wie die Basisliteratur zum wichtigsten und ausführlichsten Evangelium, nicht sehr umfangreich und auf die historische Interpretation konzentriert. Er gehörte deshalb zum Grund- und Erstbestand einer geistlichen Bibliothek des Frühmittelalters. Handschriften, die den Text enthalten, führen oft in die frühe Tätigkeit eines Skriptoriums, so der Codex Rom, Biblioteca Apostolica Vaticana, Pal. lat. 177 in insularer Majuskel aus der frühen Produktion in Lorsch. ${ }^{43}$

Neben dem im Ganzen gefestigten Entwicklungsstand der karolingischen Minuskel (bis auf ein Majuskel-N in Wortmitte fehlen die Doppelformen), der auf die Zeit nach 800 verweist, ist an dem Fragment auffällig, dass die urKürzung noch immer mit dem traditionellen $u s$-Haken ausgeführt wird, was Mitte des IX. Jahrhunderts in Mainz nur noch selten zu sein scheint. Da ein genaues Skriptorium angesichts der oben skizzierten Mainzer Vielfalt nicht benannt werden kann, gelten für das Doppelblatt Mainz, Wiss. Stadtbibliothek, Hs frag 20 folgende Angaben: Hieronymus, Commentarii in evangelium Matthaei, Mainz, 1. Drittel des IX. Jahrhunderts.

43 Becker und Licht (2016, Taf. 15).

\section{Literaturverzeichnis}

Becker, Julia; Licht, Tino (2016): Karolingische Schriftkultur: Aus der Blütezeit des Lorscher Skriptoriums. Regensburg: Schnell + Steiner.

Bierbrauer, Katharina (1997): Karolingische Buchmalerei des Maingebiets (Mainz, Würzburg). In: Kultur und Theologie: Das Frankfurter Konzil von 794. Kristallisationspunkt karolingischer Kultur. Teil 2, hg. v. Rainer Berndt, 555-70. Mainz: Selbstverl. der Ges. für Mittelrheinische Kirchengeschichte (Quellen und Abhandlungen zur mittelrheinischen Kirchengeschichte: 80).

Bischoff, Bernhard (1981): Mittelalterliche Studien: Ausgewählte Aufsätze zur Schriftkunde und Literaturgeschichte. Stuttgart: Hiersemann (Band 3).

Bischoff, Bernhard (1998): Katalog der festländischen Handschriften des neunten Jahrhunderts (mit Ausnahme der wisigotischen). Bd. 1: Aachen-Lambach. Wiesbaden: Harrassowitz (Veröffentlichungen der Kommission für die Herausgabe der mittelalterlichen Bibliothekskataloge Deutschlands und der Schweiz / Bayerische Akademie der Wissenschaften: 1).

Bischoff, Bernhard (2004): Katalog der festländischen Handschriften des neunten Jahrhunderts (mit Ausnahme der wisigotischen). Bd. 2: Laon-Paderborn. Wiesbaden: Harrassowitz (Veröffentlichungen der Kommission für die Herausgabe der mittelalterlichen Bibliothekskataloge Deutschlands und der Schweiz / Bayerische Akademie der Wissenschaften: 1).

Bischoff, Bernhard (2014): Katalog der festländischen Handschriften des neunten Jahrhunderts (mit Ausnahme der wisigotischen). Band 3: Padua-Zwickau. Wiesbaden: Harrassowitz (Veröffentlichungen der Kommission für die Herausgabe der mittelalterlichen Bibliothekskataloge Deutschlands und der Schweiz / Bayerische Akademie der Wissenschaften: 1).

Bulitta, Brigitte (2006): Ein verkanntes althochdeutsches Sprachdenkmal: die lateinisch-deutsche Beichte von Zeitz. In: Die Stiftsbibliothek und das Stiftsarchiv Zeitz : [4. Tag der Heimatgeschichte für Zeitz und Umgebung], hg. v. Detlef Deye und Roland Rittig, 47-74. Halle (Saale): Mitteldt. Verl.

Dobras, Wolfgang (2002): Eine neugefundene Quelle zur Mainzer Wirtschaftsgeschichte des 16. Jahrhunderts: die Rechnungsbücher des Mainzer Krämers Hans Drudel. In: Mainzer Zeitschrift: mittelrheinisches Jahrbuch für Archäologie, Kunst und Geschichte, 96/97, 87-114.

Engels, Renate; Doll, Ludwig Anton (2009): Palatia sacra. Teil 1: Bistum Speyer, der Archidiakonat des Dompropstes von Speyer; Bd. 1.: Die Stadt Speyer; Teil 1b: Die Kollegiatstifte s. Germani ep., ss Germani ep. et Mauritii m., ss Johannis evang. et Guidonis ab., s. Trinitatis ac omnium sanctorum. Trier: Selbstverl. der Ges. für Mittelrheinische Kirchengeschichte (Quellen und Abhandlungen zur mittelrheinischen Kirchengeschichte: 61, 1.1, 1b).

Gottlob, Theodor (1928): Der abendländische Chorepiskopat. Bonn: Schröder (Kanonistische Studien und Texte: 1).

Haebler, Konrad (1908): Makulatur-Forschung. In: Zentralblatt für Bibliothekswesen, 25, 535-44.

Hanselmann, Jan Friedrich (1987): Der Codex Vat. Pal. lat. 289. Ein Beitrag zum Mainzer Skriptorium im 9. Jahrhundert. In: Scriptorium, 41, 78-87.

Kurze, Friedrich (1891): Annales Fuldenses. Hannover (MGH: Scriptores rerum Germanicarum: 7). 
Lehnardt, Andreas; Ottermann, Annelen (2015): Fragmente jüdischer Kultur in der Stadtbibliothek Mainz: Entdeckungen und Deutungen. Mainz: Bibliotheken der Stadt Mainz (Veröffentlichungen der Bibliotheken der Stadt Mainz: 62).

Licht, Tino (2015): Aus fuldischen Handschriften: Auf der Spur des Fuldaer Hausexemplars: ein neues Hrabanfragment. In: Archiv für mittelrheinische Kirchengeschichte, 67, 401-11.

Lindsay, Wallace M.; Lehmann, Paul (1925): The (early) Mayence Scriptorium. In: Palaeographia latina, 4, 15-39.

Lowe, Elias Avery (1959): Germany: Maria-Laach - Würzburg. Oxford: Clarendon Press (Codices Latini antiquiores: a palaeographical guide to Latin mss. prior to the ninth century / ed. by Elias Avery Lowe; P. 9).

Maurus, Rabanus; Dümmler, Ernst (1899): Hrabani (Mauri) abbatis fuldensis et archiepiscopi moguntiacensis Epistolae, 379-516. Berlin (MGH: Epistolae: V).

Neuheuser, Hanns Peter; Schmitz, Wolfgang (Hrsg.) (2015): Fragment und Makulatur. Überlieferungsstörungen und Forschungsbedarf bei Kulturgut in Archiven und Bibliotheken. Wiesbaden: Harrassowitz (Buchwissenschaftliche Beiträge: 91).

Oppitz, Ulrich-Dieter (2002): Von Beutelbüchern, Pergamenteinbänden und Buchwanderungen: Beobachtungen an Handschriften und Kunstwerken aus Erfurt und anderen thüringischen Städten. In: Miszellen zur Erfurter Buch- und Bibliotheksgeschichte: Vorträge vor der Neuen Erfurter Bibliotheksgesellschaft von 1997 bis 2000. 1. Aufl., hg. v. Kathrin Paasch, 53-63. Bucha bei Jena: Quartus-Verl. (Palmbaum-Texte: 11).

Ottermann, Annelen (1998): Das Beda-Fragment Hs frag 1 in der Stadtbibliothek Mainz: Ein Beitrag zum Mainzer Skriptorium des 9. Jahrhunderts. In: Philobiblon, 42, 301-6.

Ottermann, Annelen (2008a): Qui non addit, amittit: Vom Wachsen einer Rarasammlung. In: Wolfenbütteler Notizen zur Buchgeschichte, 33, 95-107. Verfügbar unter http://www.uni-muen ster.de/Forum-Bestandserhaltung/downloads/08_Ottermann. pdf.

Ottermann, Annelen (2008b): "Rara wachsen nach": Einblicke in die Rarasammlung der Wissenschaftlichen Stadtbibliothek Mainz. Mainz: Stadt Mainz (Veröffentlichungen der Bibliotheken der Stadt Mainz: 55). Verfügbar unter http://eprints.rclis.org/bit stream/10760/17240/1/Ottermann_Rara_wachsen_nach.pdf.

Ottermann, Annelen (2015): Rezension zu Fragment und Makulatur. Überlieferungsstörungen und Forschungsbedarf bei Kulturgut in Archiven und Bibliotheken. Hg. v. Neuheuser, Hanns Peter; Schmitz, Wolfgang. In: Wolfenbütteler Notizen zur Buchgeschichte, $40(1 / 2), 148-54$.

Ottermann, Annelen (2016): Die Mainzer Karmelitenbibliothek: Spurensuche - Spurensicherung - Spurendeutung. Berlin: Logos (Berliner Arbeiten zur Bibliotheks- und Informationswissenschaft: Band 27).
Ottermann, Annelen (Hrsg.) (2014): Das spätkarolingische Fragment eines illustrierten Apokalypse-Kommentars in der Mainzer Stadtbibliothek: Bilanz einer interdisziplinären Annäherung. Mainz: Landeshaupstadt Mainz Bibliotheken der Stadt Mainz (Veröffentlichungen der Bibliotheken der Stadt Mainz: 60). Verfügbar unter http://eprints.rclis.org/28944/.

Ottermann, Annelen; Klein, Klaus (2008): Handschriftenfunde zur Literatur des Mittelalters: 185. Beitrag ; ein unbekanntes 'Rennewart'-Fragment in Mainz. In: Zeitschrift für deutsches Altertum und deutsche Literatur, 137 (3), 370-76.

Viguer, loann (1563): Institutiones ad Christianam theologiam, sacrarum, literarum, vniuersaliumque conciliorum auctoritate, necnon doctorum ecclesiasticorum eruditione confirmatae, ecclesiasticis omnibus animarum curam gerentibus admodum necessariae, opera, atque industria eruditissimi viri f. loannis Viguerii Granaten. Apud Tolosam praedicatorum Ordini adscripti, sacrae theologiae doctoris \& professoris.Titulos recens additos in sexta pagella reperies. Venetiis: ex officina Francisci Laurentini de Turino, 1563 (EDIT16 CNCE 35808). Stadtbibliothek Mainz, Sign.: XIII t: $4^{\circ} / 768 ®$.

Weiner, Andreas (1992): Die Initialornamentik der deutsch-insularen Schulen im Bereich von Fulda, Würzburg und Mainz. Würzburg: Schöningh (Quellen und Forschungen zur Geschichte des Bistums und Hochstifts Würzburg: Bd. 43).

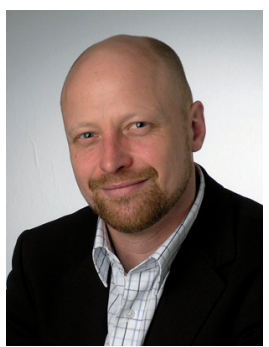

PD Dr. Tino Licht

Lateinische Philologie des Mittelalters und der Neuzeit

am Historischen Seminar

Grabengasse 3-5

D-69117 Heidelberg

tino.licht@urz.uni-heidelberg.de

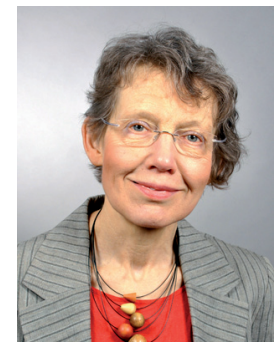

Dr. Annelen Ottermann

Wissenschaftliche Stadtbibliothek Mainz

Bereichsleitung Handschriften, Rara,

Alte Drucke und Bestandserhaltung

Rheinallee $3 \mathrm{~B}$

D-55116 Mainz

annelen.ottermann@stadt.mainz.de 